\title{
Le Systeme de Controle de la Gestion des Finances Publiques au Benin
}

\author{
Dr Sakinatou Bello*
}

\section{RESUME/ABSTRACT}

Que peut l'Etat sans les finances publiques? rien! C'est dire que les finances publiques sont au cœur de l'Etat et la raison d'être de l'Etat. Mais si elles occupent cette place centrale, c'est parce qu'elles permettent à l'Etat de se mouvoir et d'assumer les obligations qui sont les siennes à l'endroit de la communauté nationale et celle internationale. Les finances publiques ne sont rien d'autre que l'argent du peuple dont les gouvernants ont à charge la gestion. Ceci implique et impose la rigueur et la transparence dans leur gestion.

L'on ne saurait donc parler de finances publiques sans parler de gestion, de système de contrôle et d'évaluation de l'exécution effective et efficace des différentes missions en matière budgétaire. Le cas de la république du Bénin nous permet de prospecter sur ce terrain.

What can the State without public finances? nothing! This means that public finances are at the heart of the State and allows the state to exist. If Public finances such a central place, it is because they allow the State to function and to honor its obligations towards the national and international community. Public finances are nothing more than the money of the people whose management is entrusted to the government. This implies and imposes rigour and transparency in their management.

We cannot therefore talk about public finances without talking about the management, control and evaluation of the efficient and effective execution of the various budgetary tasks. The situation of the Republic of Benin is a good case study to explore the issue.

\section{INTRODUCTION}

Que peut l'Etat sans les finances publiques? Nous répondrons assurément par la négative à cette interrogation et le Professeur Michel BOUVIER justifiera notre position lorsqu'il affirme que «les finances publiques ont (...) à plusieurs reprises dans l'histoire joué un rôle majeur dans le processus de formation et de transformation de l'Etat, se situant à l'origine de la démocratie ${ }^{1}$ ». Il sera renchérit par Pierre JOXE qui disait qu' " il n'y a pas de démocratie sans finances publiques claires, sans transparence, sans règle de droit " et que

* Dr Sakinatou Bello, est enseignante chercheure à la Faculté de droit et science politique de l'université de Parakou au Bénin. Elle est actuellement la Directrice Adjointe de Cabinet du Ministre d'Etat chargé de la Plan et du Développement. E-mail : bsakinath@yahoo.fr

1 BOUVIER (M), «Réforme des finances publiques : réforme de l'Etat », RFFP, $n^{\circ} 73,2001$. 
"l'obscurité en matière de finances publiques est évidemment la source de tous les abus ou pire $»^{2}$. Mais qui parle de finances publiques parle de gestion, de système de contrôle et d'évaluation de l'exécution effective et efficace des différentes missions ${ }^{3}$ en matière budgétaire.

Du grec sustêma, un système est un ensemble d'éléments liés entre eux et formant un tout organisé pour répondre à des besoins spécifiques. Plus explicite, il est l'ensemble de pratiques, de méthodes et d'institutions formant à la fois une construction théorique et une méthode pratique.

Dérivé de la contraction des mots « contre » et « rôle » signifiant un feuillet ou un registre tenu à l'effet d'en vérifier un autre, le Professeur MEDE Nicaise estime que «le contrôle se fonde sur le souci de la régularité et $d u$ besoin de transparence des finances publiques $^{4}$ ». Cette définition renvoie au suivi traditionnel de la bonne gestion des finances publiques. Une définition exprimant l'évolution du terme contrôle en matière de maniement des deniers publics s'impose. Le terme « contrôle » renvoie d'une part, à un exercice de suivi des crédits budgétaires afin de contrôler la régularité et l'effectivité de la dépense publique et, d'autre part, à un exercice d'évaluation de la dépense publique, afin de déterminer si celle-ci a atteint les objectifs qui lui étaient assignés, et dans l'affirmative, s'il est possible d'obtenir les mêmes résultats à moindre coûts. L'évaluation essaye d'appréhender d'un point de vue principalement externe les effets et/ou la valeur de l'action considérée opération de vérification de la validité ou de la légalité.

Autrement dit, le concept dont le rôle renvoie à une opération de vérification de la validité ou de la légalité.

La notion de finances publiques est prolifique en définition et varie d'un doctrinaire à un autre et suivant des champs disciplinaires donnés. Elle serait la branche du droit public consacrée à l'étude des règles et procédures qui régissent l'activité financière des personnes publiques ainsi que les agents chargés de son exécution ${ }^{6}$. La définition d'ARK-

2 JOXE (P.), «Finances publiques : scénarios pour demain », « Introduction », in RFFP, n87, 2004, pp11-12. Cité par AHOUANKA (E), «la transparence budgétaire dans les Etats membres de l'UEMOA (Réflexion sur le nouveau rôle du parlement et de l'opinion publique), in LA LOLF dans tous ses états, Acte de colloque national des 13 et 14 février 2015, Centre des Publications Universitaire (CPU) de l'Université d'Abomey-Calavi, 2015, pp 535-563.

3 C'est un ensemble de programmes concourant à une politique publique définie et clairement identifiée par un ou plusieurs objectifs. Etienne DOUAT, "Réflexions sur la réforme de budget de l'Etat », la revue du Trésor, 86é année $\mathrm{N}^{\circ} 7$, juillet 2006.

4 MEDE (N) cité par Denagnon Marcellin TOBOSSI dans son mémoire intitulé « Contribution à l'effectivité du contrôle juridictionnel sur les finances de l'Assemblée Nationale », 2008.

5 FABIUS (L.) et MIGAUD (D.), "Contrôler réellement pour dépenser mieux et prélever moins » cité par LASCOMBE M. et VANDENDRISSCHE X., "La loi organique relative aux lois des finances et le contrôle des finances publiques », in RFAP, n¹17, 2006, p.134.

6 Boubacar Demba BA, « Finances publiques et gestion par la performance dans les pays membres de l'UEMOA : étude de cas du Sénégal », thèse de Doctorat en droit public Soutenue publiquement le lundi 30 mars 2015, 572 pages. 
WRIGHT traduit bien la notion de personnes publiques. Il définit les finances publiques comme l'étude des finances des personnes morales de droit public (Etat, collectivités territoriales, établissements publics ${ }^{7}$ ).

L'histoire des finances publiques remonte à des siècles bien avant que le parlement britannique n'arrache l'office du consentement de l'impôt au souverain au prix de luttes implacables ayant entrainé non seulement la révolution de 1688 mais aussi l'exécution du Ministre des Finances et le roi $^{8}$. La plus grande illustration francophone de la nécessité de consentement mais aussi de contrôle de l'exécution effective des contributions citoyennes est celle prévue par l'article 14 de DDHC de 1789 qui dispose que «tous les citoyens ont le droit de constater par eux-mêmes ou par leurs représentants, la nécessité de la contribution publique, de la consentir librement, d'en suivre l'emploi et d'en déterminer la quotité, l'assiette, le recouvrement et la durée ».

A l'instar d'autres pays, le Bénin a choisi l'option de la transparence dans la gestion des finances de l'Etat et des organes publics connexes, et le constituant n'a pas manqué d'enjoindre aux « citoyens chargés d'une fonction publique ou élus à une fonction politique le devoir de l'accomplir avec conscience, compétence, probité, dévouement et loyauté dans l'intérêt et le respect du bien commun " ${ }^{9}$. Mieux, la Constitution trace la voie de l'interdit d'une mauvaise gestion des deniers publics et délègue au législateur et le pouvoir judiciaire les prérogatives de protection des biens publics contre toute forme d'atteinte en ces termes : «Les biens publics sont sacrés et inviolables. Tout citoyen béninois doit les respecter scrupuleusement et les protéger. Tout acte de sabotage, de vandalisme, de corruption, de détournement, de dilapidation ou d'enrichissement illicite est réprimé dans les conditions prévues par la $l o i^{10} »$.

Même dans l'option d'une démocratie financière, le Bénin dispose d'un système de contrôle de la gestion des finances publiques caractérisé par l'existence d'un cadre législatif et institutionnel de contrôle du maniement des fonds publics. Le cadre législatif est important car nulle compétence sans texte dans un Etat de droit. À titre de mécanisme législatif, sans souci d'exhaustivité, il urge d'indexer la Constitution du 11 décembre 1990 (article 96 à 111), la loi organique 86-021 du 26 septembre 1986 relative aux Lois des Finances remplacée par la loi organique $\mathrm{N}^{\circ} 2013-14$ du 27 septembre 2013 relative aux lois de finances

7 Edward ARKWRIGHT, Jean-Luc BOEUF, Cécile COURREGES, Stanislas GODEFROY, Manuela MAGNAN, Gautier MAIGNE et Manuel VAZQUEZ, Les finances publiques et la réforme budgétaire, Paris, la Documentation française, 2009, disponible en ligne sur www.babordplus.univ-bord eaux.fr, consulté le 17 février 2019 à $23 \mathrm{~h} 37$.

8 DOUAT (E) et BADIN (X), Finances publiques, finances communautaires, nationales, sociales et locales, Paris, PUF, 3e éd., 2006, p. 6.

9 Article 35 de la Constitution du 11 décembre 1990.

10 Article 37 de la Constitution du 11 décembre 1990. 
en République du Bénin (LOLF) ${ }^{11}$, le Code de procédure civile, administrative, sociale et des comptes et les directives de l'UEMOA, régissent le domaine de la gestion des finances publiques. Le cadre institutionnel est composé des organes juridictionnels et non juridictionnels de contrôle.

Le sujet revêt un double intérêt. Il permet de faire une présentation analytique du système béninois de contrôle de la transparence et de loyauté dans la gestion des finances de 1986 à nos jours.

Quel est le visage du système de contrôle de la gestion des deniers publics au Bénin? A-t-il intégré les nouveaux modes de contrôle qui ont pour finalité la performance dans l'action publique ou est-il resté statique?

Répondre à cette problématique revient à présenter dans un premier temps le système de contrôle interne de l'administration publique sur ses agents dans sa forme rénovée ${ }^{12}$ (A) et dans un second temps, le système de contrôle externe qui a été renforcé ${ }^{13}$ (B)

\section{A- UN SYSTEME CONTROLE INTERNE RENOVÉ}

Ce système de contrôle est mis en mouvement, soit par des commis du Gouvernement (Ministère de l'Economie et des Finances et par la Présidence) pour un contrôle centralisé (I), soit par des organes dépendant de l'organisme public dépensier dans le cadre d'un contrôle décentralisé (II).

\section{I- Le contrôle centralisé : de la régularité à la performance}

Le premier organe de contrôle venant du Ministère de l'Economie et des Finances (MEF) est le Contrôle Financier (CF) qui est représenté par un délégué lui aussi fonctionnaire du MEF (DCF). Il exerce un contrôle a priori et de façon permanente sur les finances des organismes publics (notamment les ordonnateurs) assujettis aux règles de la comptabilité publique $^{14}$. A cet effet, il s'attèle à un contrôle de régularité budgétaire, du respect des règles juridiques et de l'orthodoxie financière en ce qui concerne les opérations de dépenses du budget général de l'Etat, des budgets annexes et ceux des collectivités territoriales ainsi que des budgets de certaines structures publiques désignées par arrêté du MEF. C'est à ce titre qu'au stade d'engagement de la dépense, chaque ordonnateur doit solliciter le visa du $\mathrm{CF} / \mathrm{DCF}$ pour que ce dernier puisse vérifier l'existence et la disponibilité du crédit ainsi que le chapitre correspondant à la dépense dans le respect des normes. A la phase d'ordonnan-

11 Loi organique relative aux lois de finances (LOLF), est une loi organique qui fixe le cadre juridique des lois de finances en République du Bénin. Elle est en quelque sorte une « Constitution financière $»$.

12 Article 84 alinéa 2 de la Loi organique n²013-14 du 27 septembre 2013 relative aux Lois des Finances.

13 Ibid., alinéa 5.

14 Article 87 alinéa de la Loi organique relative aux Lois des Finances. 
cement, l'ordonnateur principal soumet l'ordre de payer au contrôleur budgétaire pour l'examen de la conformité de l'engagement à l'ordonnancement. A cet effet, il vérifie la régularité de la liquidation, la régularité du service fait et la conformité du crédit alloué à celui de la demande. S'il accepte, le contrôleur appose un second visa sur la fiche. En effet, toutes les ordonnances (ordres de payer) non visées par le CF/DCF sont systématiquement rejetées par les comptables publics. ${ }^{15}$

Il assure le respect des règles de passation des marchés publics soumis à l'approbation du MEF.

Par ailleurs, il a une compétence consultative en ce qu'il donne son avis motivé sur les projets de lois, de règlements et autres mesures financières soumis à l'approbation du MEF. Dans la même veine, il est consulté par le MEF au moment de la préparation du Budget Général de l'Etat pour donner son avis sur les propositions de dépenses de l'organisme dépensier dont il assure la régularité des dépenses. Ce système mixte de contrôle est celui de la France.

A l'ère de l'épiphanie de la performance, du rôle de contrôle de la régularité sont adjoints au contrôleur financier, l'évaluation des résultats et les performances des programmes au regard des objectifs, des moyens utilisés et de l'organisation des services des ordonnateurs $^{16}$. Les contrôleurs financiers sont ainsi appelés à développer de nouveaux contrôles avec de nouvelles techniques pour de nouveaux enjeux ${ }^{17}$. Ils participent à l'identification et à la prévention des risques financiers ainsi qu'à l'analyse des facteurs explicatifs de la dépense et du coût des politiques publiques; à la vérification de la sincérité des prévisions de dépenses ainsi qu'au contrôle du document annuel de programmation budgétaire initial, des documents prévisionnels de gestion, leur modification en cours de gestion ainsi que les projets d'actes d'affectation de crédits d'engagement des dépenses. Le contrôleur financier devient un acteur de la Gestion Axée sur les Résultats (GAR). Il y a eu donc un dépassement dans le rôle classique qu'il avait hérité de la LOLF de la période révolutionnaire pour la prise en compte des exigences de la communautarisation du droit budgétaire ainsi que de l'influence des bailleurs de fonds qui y voient l'opportunité d'une meilleure information sur les résultats des actions qu'ils ont contribué à financer ${ }^{18}$.

15 C'est du moins ce qui ressort de l'article 113 du décret n²014-571 du 07 octobre 2014 portant règlement général sur la comptabilité publique : "Toute ordonnance de paiement, tout mandat de paiement ou toute délégation de crédits ne peut être présenté à la signature de l'ordonnateur qu'après avoir été soumis au visa du Contrôleur Financier ou de son Délégué. Les mandats de paiement et les délégations de crédits non revêtus du visa du contrôleur financier ou de son délégué sont nuls et de nul effet. ".

16 Article 115 du règlement général sur la comptabilité publique.

17 DJIGLA (F.L.) et AFFOUKOU (H.), « Le contrôle financier dans la réforme : statut quo ou refondation? ", in LA LOLF dans tous ses états, Acte de colloque national des 13 et 14 février 2015, Centre des Publications Universitaire (CPU) de l'Université d'Abomey-Calavi, 2015, pp 445-480.

18 D. LAMARQUE, « Les nouvelles missions du juge financier dans un contexte de budgétisation par programme », Revue béninoise de sciences juridiques et administratives $n^{\circ} 24,2011$, p. 55 . Il 
Le deuxième organe dépendant du MEF est l'Inspection Générale des Finances (IGF). Dotée d'un manuel de procédure, elle est chargée, en cours d'exécution du budget, d'une mission de surveillance et de vérification permanente de la gestion financière des ordonnateurs, des comptables ainsi que des gestionnaires d'établissements publics voire des structures autorisées aux recouvrements de fonds publics (taxes, redevances...). Pour mener à bien sa mission, elle descend inopinément dans la structure pour un contrôle sur place et peut requérir la force publique avec possibilité de prendre des mesures de sauvegarde. A la fin de son examen, elle établit un rapport soumis au principe du contradictoire, un rapport dans lequel elle fait le point de ses constats et procéder à des recommandations.

Le troisième organe est l'Inspection Générale de l'Etat (IGE). Elle est un outil de contrôle du Président de la République dont elle est rattachée directement. Elle exerce ses activités dans les mêmes conditions que l'IGF mais en cours d'exécution. Elle vérifie la gestion des services du MEF ainsi que d'autres services publics (l'Etat, les CT et les $\left.\mathrm{EPA}^{19}\right)$.

Ces deux organes spécialisés sus cités exercent un contrôle interne a posteriori sur l'exécution des lois de finances conformément à l'article 88 de la Loi organique relative aux lois de finances.

Outre le système de contrôle centralisé, des ramifications de l'IGF et l'IGE sont dans les ministères.

\section{II- Le contrôle décentralisé : de l'inspection à l'audit}

L'IGF et l'IGE ont pour béquilles, les structures sectorielles d'inspection comme l'Inspection Générale du Ministère (IGM) et l'Inspection Générale des Services (IGS). C'est la décentralisation des activités de l'IGF et de l'IGE au sein d'un Ministère. Il urge de noter que ces organes spécialisés sont créés par le décret 97- 177 du 21 avril 1977 portant réorganisation des organes de contrôle et d'inspection de l'administration publique ${ }^{20}$. L'IGM est sur trois fronts de contrôle dans un ministère : les activités techniques du ministère (1), la gestion financière et comptable (2) et la gestion administrative (3). A ce titre, la vérification de la régularité des opérations de recettes budgétaires non fiscales par les structures et organismes sous la tutelle du ministère relève de ses attributions. Vient le contrôle de régularité et d'effectivité des opérations de dépenses imputables du budget du ministère dépensier sans oublier le contrôle de l'exécution financière et physique des programmes et projets.

Quant aux IGS, elles sont installées au niveau des trois régies financières de l'Etat dont la Direction Générale des Douanes et Droits Indirects (DGDDI), la Direction Générale des Impôts et des Domaines (DGID) et la Direction Générale du Trésor et de la Comptabilité

faut noter que sous l'impulsion de la banque mondiale, le Bénin s'essaie au program budgeting depuis 2000 .

19 Etablissement public à caractère administratif.

20 L'article 4 du décret suscité énumère les différentes structures comme l'IGF, l'IGAA, l'IGSEP, l'IGSJ et l'IGE. 
Publique (DGTCP) pour assurer la légalité dans le fonctionnement des régies, le respect des règles de gestion des finances. Elle opère sur place et sur pièces des services extérieurs au trésor, des caisses intermédiaires de recettes, d'avances et de menues dépenses. Le fonctionnement de tous les services centraux est sous son contrôle ainsi que la vérification des comptes de disponibilités, de mouvements de fonds, des états de rapprochement des comptes bancaires.

En guise de petite conclusion, il faut retenir que le contrôle administratif des finances de l'Etat est exercé par nombre de structures tant centralisées que décentralisées. L'analyse $\mathrm{du}$ fonctionnement de ces unités formant le système de contrôle laisse transparaître que le contrôle est celui de la régularité juridique. Il n'intègre pas les exigences d'atteinte des objectifs fixés par la loi de finances.

Qu'en est-il du système de contrôle externe?

\section{B- UN SYSTEME DE CONTROLE EXTERNE RENFORCE}

Le contrôle des finances publiques n'est pas que propre à l'Administration. Dans la plupart des Etats francophones, des systèmes de contrôle externe sont prévus tant par la Constitution $^{21}$ que par les textes ${ }^{22}$ en vigueur. Il s'agit du contrôle parlementaire (I) et du contrôle juridictionnel (II).

\section{I- De la rationalisation du contrôle parlementaire}

A l'article 79 de la Constitution du 11 décembre 1990, le parlement reçoit le spectre pour voter la loi et consentir l'impôt. Mais la Constitution lui confère bien au-delà de cela. Le contrôle parlementaire est un contrôle politique institué par la Constitution et le Règlement Intérieur de l'Assemblée Nationale (AN), par la loi organique relative aux lois de finances et les directives communautaires. Il se fait en cours d'exécution du budget et a posteriori ${ }^{23}$. Le contrôle en cours d'exécution s'effectue par le biais des commissions d'enquête parlementaire et des questions écrites ou orales ${ }^{24}$ à l'endroit de l'exécutif. Ces commissions sont chargées du suivi et du contrôle de l'exécution des lois de finances et doivent procéder à l'évaluation de toute question relative aux finances publiques. A cet effet, elles procèdent à toutes investigations sur pièces et sur place, et à toutes auditions qu'elles jugent utiles.

L'article 119 du règlement intérieur de l'Assemblée Nationale pose les modalités d'exercice de ce type de contrôle parlementaire. A cet effet, les documents et les renseignements destinés à permettre l'exercice du contrôle du budget général de l'Etat et des budgets

21 Article 112 de la Constitution du 11 décembre 1990.

22 Article 74 de la directive 06/2009/CM/UEMOA portant loi des finances au sein de l'UEMOA.

23 Paulin Ibanda KABAKA, « Le contrôle non juridictionnel de l'exécution des dépenses publiques et du budget de l'Etat », article publié sur https://hal.archives-ouvertes.fr/hal-01283785 le 8 mars 2016 et consulté le 8 mars 2019 à $22 \mathrm{H}$.

24 Article 106 à 113 du Règlement Intérieur de l'Assemblée Nationale. 
autonomes ou la vérification des comptes des entreprises publiques et des sociétés d'économie mixtes sont communiqués par les autorités compétentes au Président de l'AN à l'attention du Président de la Commission des Finances et du rapporteur spécial désigné. Il y a lieu de s'inquiéter sur une possible "opacité » autour des travaux du rapporteur spécial, travaux qui ne peuvent faire l'objet d'information or le rôle d'information permet au parlement d'exercer un contrôle sur la politique du Gouvernement. Il se pose un problème de la vulnérabilité du contrôle. En outre, le phénomène majoritaire au niveau de l'AN peut être de nature à influencer le contrôle parlementaire de l'action du Gouvernement. Et d'ailleurs, pour pallier cet état de choses, un Protocole d'Accord fut signé entre l'AN et le PNUD pour l'installation d'une unité d'analyse, de contrôle et d'évaluation du Budget de l'Etat à l'AN le 18 janvier 2001. Les nouvelles réformes du cadre parlementaire en la matière ont permis de rationaliser le contrôle parlementaire par le rôle évaluateur ${ }^{25}$ qui est dévolue à l'institution. A cet effet, des structures d'appui furent créées pour renforcer le contrôle parlementaire de la gestion des finances publiques. Il s'agit de la Cellule d'Analyse des Politiques de Développement de l'Assemblée Nationale (CAPAN) et de l'Unité d'Analyse, de Contrôle et d'Evaluation du Budget de l'Etat (UNACEB) qui est rattachée au Secrétariat Administratif Général de l'AN. Ce partenariat a permis entre autres acquis, le renforcement des capacités des parlementaires sur les questions budgétaires, l'accès aux données produites par le Gouvernement, etc.

Le contrôle a posteriori est exercé essentiellement par le biais de la loi de règlement ${ }^{26}$. Il permet de constater le montant des encaissements en matière de recettes et de dépenses effectuées en une année. Les annexes explicatives sur l'exécution des recettes et des dépenses du budget général, du budget annexe et les comptes spéciaux du trésor ainsi que le rapport de la chambre des comptes de la Cour Suprême et de la déclaration générale de conformité, permettent d'avoir une visibilité de l'exécution du budget au cours d'une année donnée en l'occurrence l'année N-1. En cas de doute sur une information, le parlement peut demander à la juridiction des comptes toute enquête aux fins de confirmer ou d'infirmer ses doutes sur la transparence dans la gestion ${ }^{27}$.

\section{II- Au renforcement du contrôle juridictionnel}

Parmi les structures composantes du système de contrôle des finances publiques au Bénin, figure une juridiction financière, la chambre des comptes de la Cour Suprême. Elle a une double compétence. La première est de veiller à la saine gestion des fonds publics par toute structure publique (Ministères, Collectivités territoriales, entreprises publiques, services pu-

25 N. MEDE, «La nouvelle gestion budgétaire : l'expérience des budgets de programme au Bénin », Afrilex n ${ }^{\circ}$ 4, 2004, 31p.

26 Paulin Ibanda KABAKA, « Le contrôle non juridictionnel de l'exécution des dépenses publiques et du budget de l'Etat », ibid. p5.

27 Article 75 alinéa 4 de la Directive n06/2009/CM/UEMOA portant loi des finances. 
blics...etc.) et la seconde vise à informer le citoyen sur le maniement des deniers publics par les gestionnaires.

Dans l'accomplissement de la première mission, le juge financier oriente son contrôle sur la gestion des comptables publics principaux qui centralisent et justifient dans leur propre compte les opérations effectuées par leurs subordonnés car ils sont chargés de garantir le respect des textes en ce qui concerne l'exécution des recettes et l'engagement des dépenses ou de maniement de titres. On parlera donc du contrôle de régularité et de conformité puis la sanction des comptables publics. Pour ce fait, les comptables concernés présentent les comptes affirmés sincères et véritables, datés et revêtus du visa de contrôle de leur supérieur hiérarchique au juge financier (la chambre des comptes) au plus tard la fin du sixième mois suivant la clôture de l'exercice ${ }^{28}$. Dans ce contrôle objectif, le juge se prononce sur la régularité des opérations retracées dans le compte en se penchant sur la gestion patente ${ }^{29}$, la gestion de fait ${ }^{30}$ et les fautes de gestion ${ }^{31}$.

La gestion est dite patente lorsqu'elle est exercée par un comptable public. En cas de constat d'une gestion régulière, le juge financier l'atteste par un arrêt de décharge. Dans le cas contraire, un arrêt d'injonction et enjoint au comptable d'apporter la preuve de la rectification des irrégularités constatées ou de produire des justifications complémentaires ${ }^{32}$. Face aux explications et la production des pièces justificatives le déchargeant, un arrêt de levée d'injonction est rendu mais dans le cas contraire, un arrêt de débet constate le déficit et fixe le reliquat à la charge du comptable.

En ce qui concerne le contrôle de gestion de fait, le juge financier fait le constat de ce que la gestion comptable a été effectuée par toute personne n'ayant pas la qualité de comptable public ou n'agissant pas sous le contrôle de ce dernier, s'est ingéré dans le recouvrement de recettes affectées ou destinées à un organisme public ou privé doté d'un poste comptable ou dépendant d'un tel post. A cet effet, le juge déclare par un arrêt provisoire la gestion de fait et requiert une production de compte du comptable de fait dans un délai de deux mois et la procédure de vérification de la régularité suit son cours comme dans une gestion patente.

Enfin, le juge financier statut en matière de discipline financière en condamnant par une peine d'amende les comportements visés à l'article 130 de la loi n ${ }^{\circ}$ 2004-20 portant règles de procédures applicables devant les formations juridictionnelles de la Cour Suprême par tout fonctionnaire, agent civil ou militaire de l'Etat, tout agent des organismes publics et des collectivités territoriales, tout membre de cabinet ministériel ou d'une institution de la République, tout représentant, administrateur ou agent des organismes qui sont soumis au

28 Article 73 alinéa 3 de la loi $n^{\circ} 2004-20$ portant règles de procédures applicables devant les formations juridictionnelles de la Cour Suprême.

29 Ibid., article 103.

30 Ibid., article 116 et suivants.

31 Ibid., article 126.

32 Ibid., article 106. 
contrôle de la chambre des comptes, toute personne investie d'un mandat public et toute personne ayant exercé de fait lesdites fonctions.

Outre la compétence répressive du juge financier, il appui le parlement dans le contrôle de l'exécution de la loi de finances. Mieux, le juge financier réalise un audit de performance. C'est ce qui l'amène à réaliser des enquêtes relatives au maniement des deniers publics sur demande du parlement ou du Gouvernement ${ }^{33}$.

\section{Bibliographie}

Ouvrages spécifiques

AKAKPO (M. B.), « Démocratie financière en Afrique occidentale francophone », FES-BENIN, Août $2015,167 \mathrm{p}$.

DOUAT (E) et BADIN (X), Finances publiques, finances communautaires, nationales, sociales et locales, Paris, PUF, 3e éd., 2006, 485 p.

\section{Thèses et mémoires}

EKOBENA (J-M. O.), "les démarches de modernisation du système camerounais de contrôle des finances publiques », Mémoire de Master II, Ecole Normale d'Administration de l'Université de Strasbourg, 21 mai 2013, $100 \mathrm{p}$.

BA (B. D.), «Finances publiques et gestion par la performance dans les pays membres de l'UEMOA : étude de cas du Sénégal », thèse de Doctorat en droit public Soutenue publiquement le lundi 30 mars 2015, $572 \mathrm{p}$.

YOSSUNDARA (A.), "Le contrôle des finances publiques en Thailande », Thèse de Doctorat, Université Paris Panthéon Sorbonne, présentée et soutenue le 17 septembre 2013, 552 p.

\section{Articles}

AHOUANKA (E), « la transparence budgétaire dans les Etats membres de l'UEMOA (Réflexion sur le nouveau rôle du parlement et de l'opinion publique), in LA LOLF dans tous ses états, Acte de colloque national des 13 et 14 février 2015, Centre des Publications Universitaire (CPU) de l'Université d'Abomey-Calavi, 2015, 2015, pp 535-563.

AKAKPO (M.), "Le contrôle de performance : comment et avec qui? », in LA LOLF dans tous ses états, Acte de colloque national des 13 et 14 février 2015, Centre des Publications Universitaire (CPU) de l'Université d'Abomey-Calavi, 2015, pp 153-168.

DJIGLA (F.L.) et AFFOUKOU (H.), « Le contrôle financier dans la réforme : statut quo ou refondation? ", in LA LOLF dans tous ses états, Acte de colloque national des 13 et 14 février 2015, Centre des Publications Universitaire (CPU) de l'Université d'Abomey-Calavi, 2015, p. 453.

33 N. MEDE, «La nouvelle gestion budgétaire : l'expérience des budgets de programme au Bénin », ibid. p.26. 
FABIUS (L.) et MIGAUD (D.), "Contrôler réellement pour dépenser mieux et prélever moins » cité par LASCOMBE M. et VANDENDRISSCHE X., " La loi organique relative aux lois des finances et le contrôle des finances publiques », in RFAP, $\mathrm{n}^{\circ} 117,2006$, p. 134.

HOMEGNON (N.), "Le PAP et le RAP : une évaluation ex ante » », in LA LOLF dans tous ses états, Acte de colloque national des 13 et 14 février 2015, pp 481-501.

KABAKA (P. I.), « Le contrôle non juridictionnel de l'exécution des dépenses publiques et du budget de l'Etat », article publié sur https://hal.archives-ouvertes.fr/hal-01283785 le 8 mars 2016 et consulté le 8 mars 2019 à $22 \mathrm{H}$.

$\operatorname{MEDE}(N$.$) , «La nouvelle gestion budgétaire : l'expérience des budgets de programme au Bénin »,$ Afrilex n ${ }^{\circ}$, 2004, $31 \mathrm{p}$.

LAMARQUE (D.) «Les nouvelles missions du juge financier dans un contexte de budgétisation par programme », Revue béninoise de sciences juridiques et administratives $n^{\circ} 24,2011$.

TONI (E.), « La loi organique relative aux lois des finances : 25 ans après », $13 \mathrm{p}$.

TOURE (C.M.), « le système de contrôle des finances publiques au Mali », Afrilex, n4, pp150-170.

\section{Textes juridiques}

Loi n ${ }^{\circ 0-32 ~ d u ~} 11$ décembre 1990 portant Constitution de la République du Bénin.

Loi n ${ }^{\circ} 86-021$ du 26 septembre 1986 portant loi organique relative aux lois des finances.

Loi Organique n²013-14 du 27 septembre 2013 relative aux Lois des Finances.

loi n²004-20 du 17 août 2007 portant règles de procédures applicables devant les formations juridictionnelles de la Cour Suprême.

Directive $\mathrm{n}^{\circ}$ 06/2009/CM/UEMOA portant lois de finances au sein de l'UEMOA;

Directives $n^{\circ} 01 / 2009 / \mathrm{CM} /$ UEMOA portant Code de transparence dans la gestion des finances publiques au sein de l'UEMOA.

Décret n²015-035 du 29 janvier 2015 portant Code de Transparence dans la gestion des finances publiques en République du Bénin. 